\title{
Facile Construction and Divergent Transformation of Polycyclic Isoxazoles: Direct Access to Polyketide Architectures
}

\author{
Jeffrey W. Bode, Yoshifumi Hachisu, Tomoo Matsuura and Keisuke Suzuki \\ Department of Chemistry, Tokyo Institute of Technology, and CREST, Japan Science and \\ Technology (JST) Corporation, O-okayama, Meguro-ku, Tokyo 152-8551, Japan
}

\section{Supporting Information}

General Methods. All reactions utilizing air- or moisture-sensitive reagents were performed in dried glassware under an atmosphere of dry $\mathrm{N}_{2}$. EtOH, MeOH, and $\mathrm{PrOH}$ were distilled from $\mathrm{Na}$ and stored over $4 \AA$ MS. Commercially available anhydrous, stabilizer-free THF (Kanto No. 41001) and $\mathrm{Et}_{2} \mathrm{O}$ (Kanto No. 14547-95) were used without further purification. Diisopropylamine (DIPA), and triethylamine $\left(\mathrm{NEt}_{3}\right)$ were distilled from $\mathrm{KOH}$ and stored over $4 \AA$ MS. Other reagents were used without further purification. Thin layer chromatography (TLC) was performed on Merck precoated plates (silica gel $60 \mathrm{~F}_{254}$, Art 5715, $0.25 \mathrm{~mm}$ ) and were visualized by fluorescence quenching under UV light or by staining with phosphomolybdic acid. Silica-gel preparative thin-layer chromatography (PTLC) was performed using plates prepared from Merck Kieselgel $60 \mathrm{PF}_{254}$ (Art 7747). Column chromatography was performed on E. Merck Silica Gel 60 (230-400 Mesh) using a forced flow of 0.5-1.0 bar. Melting point (mp) determinations were performed using a Yanaco MP-S3 instrument and are uncorrected. ${ }^{1} \mathrm{H}$ NMR (400 MHz) and ${ }^{13} \mathrm{C}$ NMR (100 MHz) were measured on a JEOL JMN Lambda-400 spectrometer. Chemical shifts are expressed in parts per million (PPM) downfield from internal tetramethylsilane $(\square=0)$ and coupling constants are reported as hertz $(\mathrm{Hz})$. Splitting patterns are indicated as follows: br, broad; s, singlet; $d$, doublet,; t, triplet; q, quartet; $\mathrm{m}$, multiplet. Infrared (IR) spectra were recorded on a Jasco IRA-202 spectrometer and are reported as wavenumbers $\left(\mathrm{cm}^{-1}\right)$. 


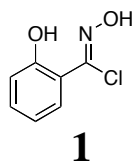

General Procedure for Oxime Chlorination. To a solution of salicylaldoxime (4.23 g, $30.8 \mathrm{mmol}$ ) in $\mathrm{CHCl}_{3}(29 \mathrm{~mL})$ was added pyridine ( $\left.243 \mathrm{mg}, 3.07 \mathrm{mmol}\right)$. The temperature was raised to $40{ }^{\circ} \mathrm{C}$ and $N$-chlorosuccinimide ( $4.53 \mathrm{~g}, 33.9 \mathrm{mmol}$ ) was added to the resulting solution. Stirring was continued at this temperature for $3 \mathrm{~h}$ before the reaction mixture was diluted by $\mathrm{CH}_{2} \mathrm{Cl}_{2}(200 \mathrm{~mL})$. The organic layer was washed by water ( 2 X $100 \mathrm{~mL}$ ) and brine, dried over $\mathrm{Na}_{2} \mathrm{SO}_{4}$, and concentrated under reduced pressure to afford hydoxyiminoyl chloride $1(5.05 \mathrm{~g}, 95 \%,>95 \%$ purity) as light yellow powder.

${ }^{1} \mathbf{H}$ NMR $\left(\mathrm{CDCl}_{3}, 400 \mathrm{MHz}\right) \square 10.04(\mathrm{~s}, 1 \mathrm{H}), 8.07$ (s, $\left.1 \mathrm{H}\right), 7.82(\mathrm{dd}, 1 \mathrm{H}, J=8.3,1.4)$, 7.35 (dd, $1 \mathrm{H}, J=8.3,7.9), 6.96-7.04(\mathrm{~m}, 2 \mathrm{H})$

${ }^{13} \mathbf{C}$ NMR $\left(\mathrm{CDCl}_{3}, 400 \mathrm{MHz}\right) \square 156.3,142.9,132.3,129.4,119.9,117.1,115.7$

IR (thin film) $\square 3305,1608,1584,1475,1413,1243,1160,944,758$

Anal. Calc'd for $\mathrm{C}_{7} \mathrm{H}_{6} \mathrm{ClNO}_{2} ; \mathrm{C}, 49.00 ; \mathrm{H}, 3.52 ; \mathrm{N}, 8.17$. Found: C, 48.74; H, 3.70; N, 8.08

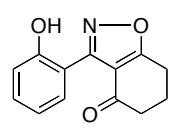

3

General Procedure for Isoxazole Formation: To a solution of sodium isoproxide, prepared from $0.045 \mathrm{~g}$ of $\mathrm{Na}$ in $10 \mathrm{~mL}$ of isopropyl alcohol, was added 1,3cyclohexanedione (2a) $(0.219 \mathrm{~g}, 1.96 \mathrm{mmol})$ and hydroximinoyl chloride $\mathbf{1}(0.161 \mathrm{~g}$, $0.937 \mathrm{mmol}$ ) successively. After stirring $30 \mathrm{~min}$ at $\mathrm{rt}$, the reaction mixture was poured into water, and extracted with ethyl acetate $(3 \times 15 \mathrm{~mL})$. The combined organic layer was washed with brine, dried over $\mathrm{Na}_{2} \mathrm{SO}_{4}$, and concentrated under reduced pressure. The crude product was purified by flash chromatography to provide isoxazole $\mathbf{3}$ as a white solid (0.162 g, 76\% yield). 
${ }^{1} \mathbf{H}$ NMR $\left(\mathrm{CDCl}_{3}, 400 \mathrm{MHz}\right) \square 9.46$ (br s, $\left.1 \mathrm{H}\right), 8.57$ (dd, $\left.1 \mathrm{H}, J=8.0,1.7\right), 7.37$ (ddd, 1 $\mathrm{H}, J=8.3,7.3,1.7), 7.05$ (dd, $1 \mathrm{H}, J=7.3,1.5), 7.01$ (ddd, $1 \mathrm{H}, J=8.3,8.0,1.5), 3.04$ $(\mathrm{dd}, 2 \mathrm{H}, J=6.5,6.3), 2.61(\mathrm{dd}, 2 \mathrm{H}, J=7.0,6.6), 2.30-2.23(\mathrm{~m}, 2 \mathrm{H})$

${ }^{13}$ C NMR $\left(100 \mathrm{MHz}, \mathrm{CDCl}_{3}\right) \square 192.1,181.6,159.3,156.5,132.3,131.8,119.7,117.3$, $114.6,112.8,38.8,23.2,21.4$

IR (KBr) $\square 3146,1684,1586,1568,1497,1485,1449,1429,1395,1352,1297,1237$, 1020, 762, 658, 596

Anal. calc'd for $\mathrm{C}_{13} \mathrm{H}_{11} \mathrm{NO}_{3}$ : C, 68.11; H 4.84; N, 6.11; found, C, 67.92; H, 4.82; N, 5.94. m.p. $107.3-110.7^{\circ} \mathrm{C}$

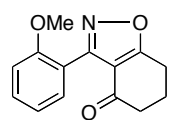

8

${ }^{1} \mathbf{H}$ NMR $\left(\mathrm{CDCl}_{3}, 400 \mathrm{MHz}\right) \square 7.45(\mathrm{ddd}, 1 \mathrm{H}, J=1.8,6.4,8.5), 7.41(\mathrm{dd}, 1 \mathrm{H}, J=7.3$, 1.8), 7.02 (dd, $1 \mathrm{H}, J=7.3,6.4), 7.00(\mathrm{~d}, 1 \mathrm{H}, J=8.5), 3.79$ (s, $3 \mathrm{H}), 3.06$ (t, $2 \mathrm{H}, J=$ 6.2), $2.51(\mathrm{dd}, 2 \mathrm{H}, J=5.5,7.0), 2.30-2.25(\mathrm{~m}, 2 \mathrm{H})$

${ }^{13}$ C NMR $\left(100 \mathrm{MHz}, \mathrm{CDCl}_{3}\right) \square 191.3,180.6,157.9,157.5,131.6,130.6,120.4,116.8$, $115.6,111.2,55.7,38.3,23.3,22.2$

IR (KBr) $\square 2691,2839,1684,1607,1474,1435,1280,1255,1017,756$

Anal. calc'd for $\mathrm{C}_{14} \mathrm{H}_{13} \mathrm{NO}_{3}$ : C, 69.12; H 5.39; N, 5.76; found, C, 69.29; H, 5.53; N, 5.58. M.P. $95.1-96.0^{\circ} \mathrm{C}$

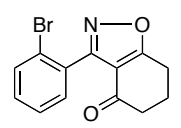

9

${ }^{1} \mathbf{H}$ NMR $\left(\mathrm{CDCl}_{3}, 400 \mathrm{MHz}\right) \square 7.68(\mathrm{~d}, 1 \mathrm{H}, J=7.8), 7.30-7.45(\mathrm{~m}, 3 \mathrm{H}), 3.10(\mathrm{t}, 2 \mathrm{H}, J=$ 6.4), 2.54 (t, $2 \mathrm{H}, J=6.4), 2.23-2.33(\mathrm{~m}, 2 \mathrm{H})$

${ }^{13} \mathbf{C ~ N M R}\left(\mathrm{CDCl}_{3}, 100 \mathrm{MHz}\right) \square 191.3,180.9,159.3,133.0,131.3,131.2,129.1,123.3$, $115.3,112.7,38.1,23.3,22.2$

IR (thin film) $\square 3061,2982,2928,2868,1689,1585,1460,1348,1062,1014,887,844$, 748,596

Anal. Calc'd for $\mathrm{C}_{13} \mathrm{H}_{10} \mathrm{BrNO}_{2}$ : C, 53.45; H, 3.45; N, 4.80. Found: C, 53.63; H, 3.67; N, 4.56

M.P. $99^{\circ} \mathrm{C}$

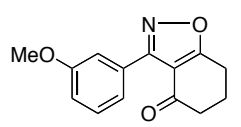

10 
${ }^{1} \mathbf{H}$ NMR $\left(\mathrm{CDCl}_{3}, 400 \mathrm{MHz}\right) \square 7.71(\mathrm{dd}, 1 \mathrm{H}, J=2.5,1.5), 7.66(\mathrm{ddd}, 1 \mathrm{H}, J=8.0,1.5$, $0.6 \mathrm{~Hz}$ ), 7.37 (t, $1 \mathrm{H}, J=8.0$ ), 7.33 (ddd, $1 \mathrm{H}, J=8.0,2.5,0.6), 3.88$ (s, $3 \mathrm{H}), 3.07$ (t, $2 \mathrm{H}$, $J=6.6), 2.61(\mathrm{t}, 2 \mathrm{H}, J=6.6), 2.30-2.23(\mathrm{~m}, 2 \mathrm{H})$

${ }^{13} \mathbf{C ~ N M R}\left(\mathrm{CDCl}_{3}, 100 \mathrm{~Hz}\right) \square 192.0,182.2,159.8,159.4,129.4,128.5,117.1,114.1$, $113.9,121.4,55.3,38.8,23.4,21.9$

IR (thin film) $\square 3084,2957,1688,1605,1574,1472,1435,1242,1047,1018,894,830$, 793, 690, 594

Anal. Calc'd for $\mathrm{C}_{14} \mathrm{H}_{13} \mathrm{NO}_{3}$ : C, 69.12; H, 5.39; N, 5.76. Found: C, 69.08; H, 5.35; N, 5.47 .

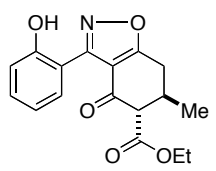

11

\section{General procedure for the reaction of isolated diketone salts in tolune}

To a suspension of 7 (438 $\mathrm{mg}, 1.99 \mathrm{mmol})$ in toluene $(5 \mathrm{~mL})$ was added hydroximinoyl chloride 1 (172 mg, $1.00 \mathrm{mmol})$ in toluene $(5 \mathrm{~mL})$ at ambient temperature. After stirring for $30 \mathrm{~min}$, the reaction mixture was poured into water and the products were extracted with EtOAc ( 3 x $10 \mathrm{~mL})$. The extracts were washed by $\mathrm{H}_{2} \mathrm{O}$ and brine, dried over $\mathrm{Na}_{2} \mathrm{SO}_{4}$, and evaporated under reduced pressure. The residue was purified by flash chromatography (25\% EtOAc/Hexane) to give $\mathbf{1 1}$ as a white solid (221 mg, 70\%).

${ }^{1} \mathbf{H}$ NMR $\left(\mathrm{CDCl}_{3}, 400 \mathrm{MHz}\right) \square 9.38$ (br s, $\left.1 \mathrm{H}\right), 8.57$ (dd, $\left.1 \mathrm{H}, J=7.3,0.7\right), 7.38$ (t, $1 \mathrm{H}$, $J=7.3$ ), 7.07-6.99 (m, $2 \mathrm{H}), 4.29$ (q, $2 \mathrm{H}, J=7.0), 3.38-3.31$ (m, $2 \mathrm{H}), 2.94-2.85$ (m, 1 $\mathrm{H}), 2.80(\mathrm{dd}, 1 \mathrm{H}, J=17.8,9.8), 1.32(\mathrm{t}, 3 \mathrm{H}, J=7.0), 1.25(\mathrm{~d}, 3 \mathrm{H}, J=6.6)$

${ }^{13} \mathbf{C}$ NMR $\left(100 \mathrm{MHz}, \mathrm{CDCl}_{3}\right) \square 187.0,180.4,159.7,169.0,156.6,132.6,131.8,119.8$, $117.3,113.7,112.2,62.6,61.7,32.5,29.7,19.6,14.2$

IR (thin film) $\square 3230(\mathrm{OH}), 2974,1739,1691,1590,1482,1253,1024$

Anal. calc'd for $\mathrm{C}_{17} \mathrm{H}_{17} \mathrm{NO}_{5}, \mathrm{C} 64.75, \mathrm{H}, 5.43, \mathrm{~N}, 4.44$; found $\mathrm{C} 64.53, \mathrm{H}$ 5.45, N 4.25 MP $118-119^{\circ} \mathrm{C}$

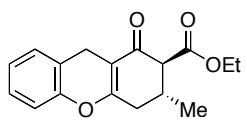

14

${ }^{1} \mathbf{H}$ NMR $\left(\mathrm{CDCl}_{3}, 400 \mathrm{MHz}\right) \square 7.19-7.10$ (m, $\left.2 \mathrm{H}\right), 7.05$ (ddd, $\left.1 \mathrm{H}, J=0.7,7.0,7.6\right), 6.95$ (dd, $1 \mathrm{H}, J=0.7,8.2), 4.33-4.21(\mathrm{~m}, 2 \mathrm{H}), 3.48$ (br s, $2 \mathrm{H}), 3.11(\mathrm{~d}, 1 \mathrm{H}, J=11.1), 2.61$ 2.69 (m, 2 H), 2.34 (br dd, $1 \mathrm{H}, J=9.7,18.6), 1.31$ (t, $3 \mathrm{H}, J=7.1), 1.13$ (d, $3 \mathrm{H}, J=6.3$ ) 
${ }^{13}$ C NMR $\left(100 \mathrm{MHz}, \mathrm{CDCl}_{3}\right) \square 192.8,170.0,165.7,149.5,129.6,127.6,124.7,120.4$, $116.4,109.0,61.0,60.4,34.4,30.8,21.0,19.5,14.1$

IR $(\mathrm{KBr}) \square 2963,1734,1636,1582,1495,1459,1396,1315,1235,1196,1176$, 1146, 1089, 1008, 768

Anal. calc'd for $\mathrm{C}_{17} \mathrm{H}_{18} \mathrm{O}_{4}$ : C, 71.31; H, 6.34; found $\mathrm{C} 71.14 ; \mathrm{H}, 6.26$

M.P. $91.0-94.2{ }^{\circ} \mathrm{C}$

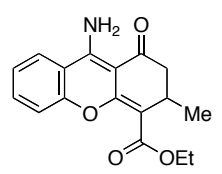

15

${ }^{1} \mathbf{H}$ NMR $\left(\mathrm{CD}_{3} \mathrm{OD}, 400 \mathrm{MHz}\right) \square 10.61-10.50$ (br s, $\left.1 \mathrm{H}\right), 7.76(\mathrm{~d}, 1 \mathrm{H}, J=8.0), 7.43$ (dd, $1 \mathrm{H}, J=7.2,8.2), 7.11$ (d, $1 \mathrm{H}, J=8.2$ ), 7.09 (dd, $1 \mathrm{H}, J=7.2,8.0), 6.02-5.83$ (br s, $1 \mathrm{H}$ ), 4.11 (q, $2 \mathrm{H}, J=7.0), 3.00-2.93(\mathrm{~m}, 1 \mathrm{H}), 2.68$ (dd, $1 \mathrm{H}, J=6.8,15.7), 2.16$ (dd, $1 \mathrm{H}, J=$ $1.9,15.7), 1.24$ (t, $3 \mathrm{H}, J=7.0), 0.93$ (d, $3 \mathrm{H}, J=6.8$ )

${ }^{13}$ C NMR $\left(100 \mathrm{MHz}, \mathrm{CD}_{3} \mathrm{OD}\right) \square 199.7,168.4,159.0,155.4,152.7,135.3,124.7,124.5$, $118.3,115.3,97.4,96.8,60.6,48.4,45.4,28.5,14.9$

IR (KBr) $\square 2963,1734,1636,1582,1494,1459,1396,1315,1235,1196,1176,1146$, 1089, 1008, 768

Anal. calc'd for $\mathrm{C}_{17} \mathrm{H}_{17} \mathrm{NO}_{4}$ : C, 68.21; H, 5.72; N, 4.68; found C, 67.97; H, 5.54; N, 4.53

M.P. $167.6-174.1^{\circ} \mathrm{C}$

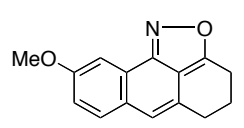

18

${ }^{1} \mathbf{H}$ NMR $\left(\mathrm{CDCl}_{3}, 400 \mathrm{MHz}\right) \square * 7.99(\mathrm{~d}, 1 \mathrm{H}, J=8.0), 7.81(\mathrm{~d}, 1 \mathrm{H}, J=2.4), 7.59(\mathrm{~d}, 1 \mathrm{H}$, $J=8.8)$, *7.45 (dd, $1 \mathrm{H}, J=8.0,8.0)$, *7.37 (s, $1 \mathrm{H}), 7.19(\mathrm{dd}, 1 \mathrm{H}, J=8.8,2.4)$, *7.07 (d, $1 \mathrm{H}, J=8.0), 6.80(\mathrm{~s}, 1 \mathrm{H}), * 3.99(\mathrm{~s}, 3 \mathrm{H}), 3.95(\mathrm{~s}, 3 \mathrm{H}), 3.14(\mathrm{t}, 2 \mathrm{H}, J=6.0), 2.88(\mathrm{t}, 2$ $\mathrm{H}, J=6.0), 2.26-2.19(\mathrm{~m}, 2 \mathrm{H})$ (* denotes minor positional isomer peaks)

${ }^{13} \mathbf{C}$ NMR $\left(\mathrm{CDCl}_{3}, 400 \mathrm{MHz}\right) \square 168.2,158.0,154.6,130.7,129.9,127.7,122.0,119.1$, $118.3,116.0,106.2,55.6,25.8,24.4,23.8$

IR (thin film) $\square 2942,2834,1662,1598,1520,1471,1435,1328,1240,1109,1032,883$, 843,794 


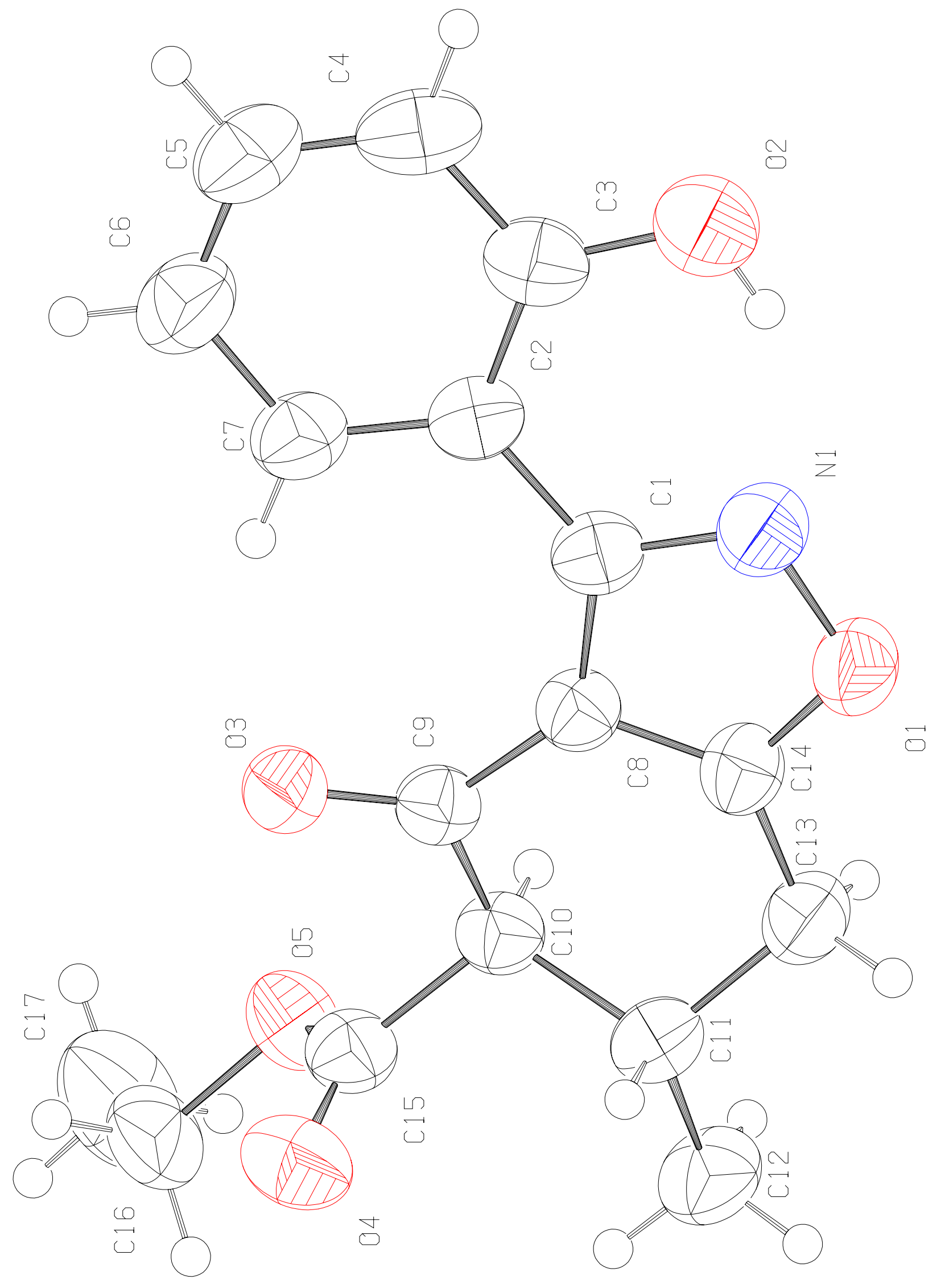

